\title{
Antifungals: from genomics to resistance and the development of novel
} agents. Edited by Alix T. Coste and Patrick Vandeputte. 2015. ISBN 978-1910190-01-2 (hdbk), 978-1-1910910-02-9 (ebk). Pp. ix + 334, illustr. (some col.), Caister: Caister Academic Press. Price: $f$ 159, US\$ 319 (hdlbk or ebk)

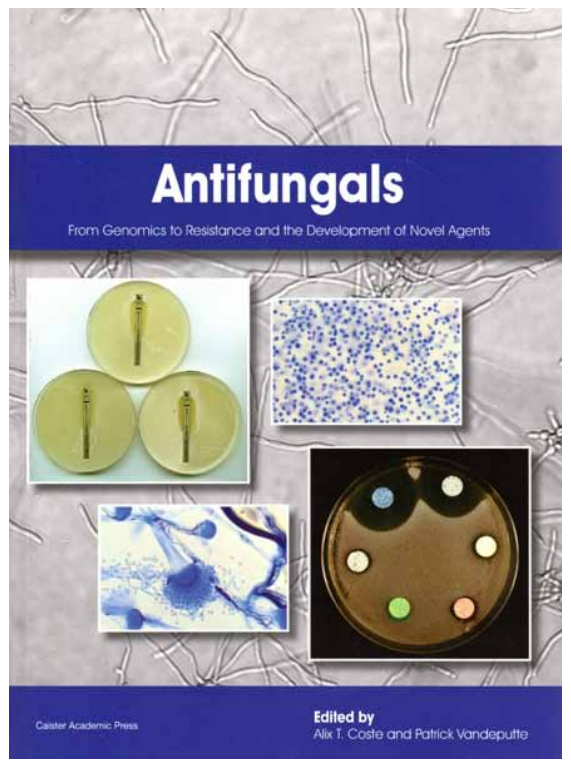

There are relatively few antifungal drugs available for clinical use and issues of drug resistance, so the search for novel ones is a priority. This is especially so as the Preface indicates that systemic fungal infections have mortality rates of to $50 \%$. The increasing use of immunosuppressant therapies has led to the widespread use of antifungal drugs and has contributed to the rise of resistance in fungi, just as has the overuse of antibacterial antibiotics to drug resistance in bacteria. This book provides a valuable background to the different approaches now becoming available. Twenty-nine authors drawn from 11 countries have combined to produce this timely and scholarly overview.

The topics covered are wide-ranging, starting with chapters on the molecular basis of resistance in Aspergillus fumigatus, Candida, and other fungi. The relatively recently discovered endocandins (which include caspofungin) seem especially promising, acting by inhibiting cell-wall synthesis, but some fungi have intrinsic resistance and mutations can also lead to resistance. Fungal biofilms are a special problem as they can affect nearly every organ and are inherently resistant to antifungals. The use of combinations of drugs has some promise, for example fluconazole and milbemycin, and there is an "Antifungal Synergistic Drug Database" which displays outcomes as a "cytoscope". Already discovered compounds in collections but which were not known to have value as antifungals are also being evaluated, and I was interested to see that acetylsalicylic acid had emerged as one with promise.

The possibility of new targets for antifungals is also explored, to take advantage of recent discoveries in fungal biology, notably $\mathrm{CO}_{2}$ sensing, quorum sensing, cell wall biogenesis, and intracellular proliferation. New technology is also leading to new horizons in largescale antifungal drug candidate "target deconvolution" (i.e. characterization of the mode of action). This is being achieved through, for example, the use of barcode and protein microarrays and transcriptome analysis, high content screening using highthroughput image capture, and automated microscopy. The genome of Candida albicans is particularly well-known, and in this case the genome re-arrangements that occur following exposure to antifungals and lead to resistance are coming to light. Another approach to limiting fungal infections is to modulate the host response, with a view to the eventual development of antifungal immunotherapies. Improved understanding of host-fungus interactions, however, is needed if antifungal vaccines are ever to be designed. Animal models are a prerequisite for clinical trials with candidate antifungals, but it is, nevertheless, acknowledged that the perfect animal model probably does not exist.

The book is well presented with informative, often coloured, explanatory diagrams, and an index which includes all mentions of particular compounds. The editors are to be congratulated on the selection of topics and contributors to produce such an instructive volume in such a fast-moving and topical field.

\section{Hidden Histories and Ancient Mysteries of Witches, Plants, and Fungi. By Frank B. Dugan. 2015. ISBN 978-0-89054-465-5. Pp. ix + 180, illustr. St Paul, MN: American Phytopathological Society Press. Price: US \$ 69.95.}

Dugan is already well-known for his research and documentation of traditional uses of fungi and their roles in plant diseases (e.g. Dugan 2008, 2011). That on ethnomycology was covered in IMA Fungus 3 (1): (36)-(37) (June 2012). The focus in this new book is plants, and especially crop plants, but what is of most interest to mycologists here is the critical position fungi have played in shaping agriculture, particularly in the Old World. The interpretation of early texts is analyzed etymologically, and it emerges that devastating fungal diseases of cereal crops may well have been a spur to the increased consumption of legume crops in Europe. Vetches, lentils, and peas became foods, particularly for livestock and the poor, and in times of shortage these were made into breads despite the major issues of toxicity. Ascochyta species on the legumes, however, appear to have caused major disease problems themselves and changes in how the crops were grown.

As in his last book, the role of women as the guardians of the knowledge of medicinal uses of plants, and to a lesser extent fungi, is emphasized. Here there is a whole chapter on "cunning women", documented by archaeological and linguistic evidence as well as herbals, and another on "kitchen witches" in which the role of herb gardens features strongly. Other chapters concern particular crops, especially North American, and links to customs and religion.

The book is in a most readable style, and can be opened almost anywhere and dipped into as it is packed with fascinating tid-bits, including some extensive and often scholarly footnotes. There are also intriguing well-annotated illustrations, including early paintings and engravings. The depth of research this has involved is impressive, something reflected in the "Literature cited" section which occupies 


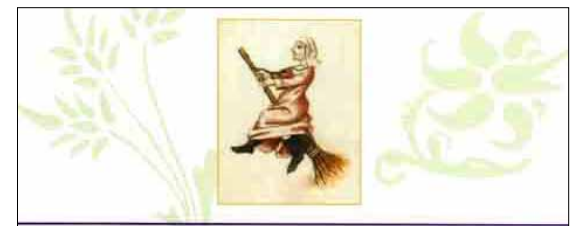

\section{Hidden Histories and Ancient Mysteries of Witches, Plants, and Fungi}

Frank Dugan

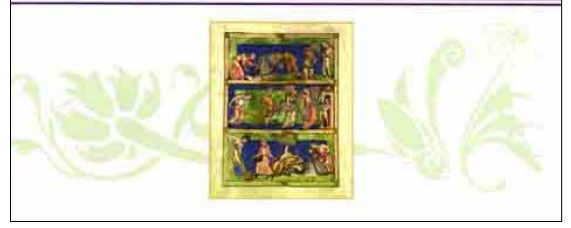

31 pages in a smaller type - about one sixth of the whole book. This is an enjoyable and fascinating read for mycologists interested in ethnomycology, and also for plant pathologists wishing to broaden their horizons.

Dugan FM (2008) Fungi in the Ancient World:

how mushrooms, mildews, moulds, and yeast shaped the early civilisations of Europe, the Mediterranean, and the Near East. St Paul, MN:

American Phytopathological Society Press.

Dugan FM (2011) Conspectus of World

Ethnomycology: fungi in ceremonies, crafts,

medicines, and myths. St Paul, MN: American

Phytopathological Society Press.

\section{Systematics and Evolution. Part B. Edited by David J. McLaughlin and Joseph W. Spatafora. 2015. ISBN 978-3-662-46010-8 (hdbk), 978-3-662-46011-5 (ebk). Heidelberg: Springer. [The Mycota Vol. 7B, 2nd edn.] Pp. xxiii + 311, illustr. Price: US \$ 199 (holbk), US\$ 159 (ebk).}

Part A of the second edition of this volume appeared last year (see IMA Fungus 5 (2): (55)-(56), December 2014), and was concerned with fungal-like organisms (Acrasiomycota, Dictyosteliomycota, and Myxomycota; Labyrinthulomycota, Hyphochytriomycota, and Oomycota; and Phytomyxea) and the fungal phyla Basidiomycota, Blastocladiomycota, Chytridiomycota, Entomophthoromycota, Glomeromycota, Neocalligmastigomycota, Microsporidia, and Monoblepharidiomycota. The present Part, in contrast, has just over half devoted to Ascomycota, and then chapters addressing more general topics.

The progress made in understanding relationships within Ascomycota over the 14 years since the first edition is immediately apparent from the dominance of phylogenetic trees. I was, however, pleased to see that the molecular data was used as the framework, but that there were accounts of the features of orders (and in some cases families), generally accompanied by line drawings, but sadly with rather few photographs, though a few are in colour. More photographs would have added much to the appeal of the book. One chapter covers ascomycete yeasts and yeast-like fungi (Saccharomycotina and Taphrinomycotina), which is followed by separate chapters on: Pezizomycetes and Orbiliomycetes; Sordariomycetes and Leotiomycetes; Lecanoromycetes; Eurotiomycetes; and Dothideomycetes and Arthoniomycetes. The coverage of the lichenized groups is especially welcome as they failed to make it into the first edition and modern synopses of many orders in those classes have been lacking. Sadly, however, the opportunity to have keys even down to order was missed.

A section on "Nomenclature and Documentation" includes a rather good overview of what has been happening in fungal nomenclature, and potential changes that may be expected. Most welcome is a series of practical notes on using the International Code of Nomenclature for algae, fungi, and plants presented as responses to a series of "How do I ... " questions. This will be very useful for those wrestling with the complexities of the Code. Pragmatism is also to the fore in the following contribution on herbaria and culture collections, though I would have thought the terms "fungaria and biological resource centres" might have been more in keeping with mycology today. There is practical guidance on specimen preservation, labeling, borrowing, and obtaining molecular data from specimens, followed by an overview of different methods of culture preservation and their relative merits.

The final section of this Part, dubbed "Evolution", has three very different chapters, all of which merit considerable attention. These address subcellular structure and biochemical features in fungal phylogeny, fungal diversity in the fossil record (a primer for Taylor et al. 2015, featured below), and phylogenomics - something which is sure to become
Edited by

Karl Esser

THE MYCOTA

A Comprehensive Treatise on Fungi as Experimental Systems for Basic and Applied Research

Systematics and Evolution Part B VII

Second Edition

David J. McLaughlin

JosephW. Spatafora

Volume Editors

Q) Springer

prominent in any third edition a decade or so hence.

The whole is well-produced, with the practice of picking out key points in the text in bold continued, and the halftone photographs are crisper than in Part A. The editors are to be congratulated on completing this new overview of the diversity and relationships of fungi and fungus-like organisms, which merits a place as a reference work wherever mycology is taught.

Taylor TN, Krings M, Taylor EL (2015) Fossil Fungi.

Amsterdam: Academic Press. 
Lichen Secondary Metabolites: bioactive properties and pharmaceutical potential. By Branislav Ranković B. 2015. ISBN 978-3-319-13373-7 (hdbk), 9783-319-13374-4 (ebk). Pp. v + 202, illustr. (some col.). Cham: Springer International Publishing. Price: $f 126$ (hdbk); $£$ 110.50, 118,99 € or US\$ 149 (ebk).

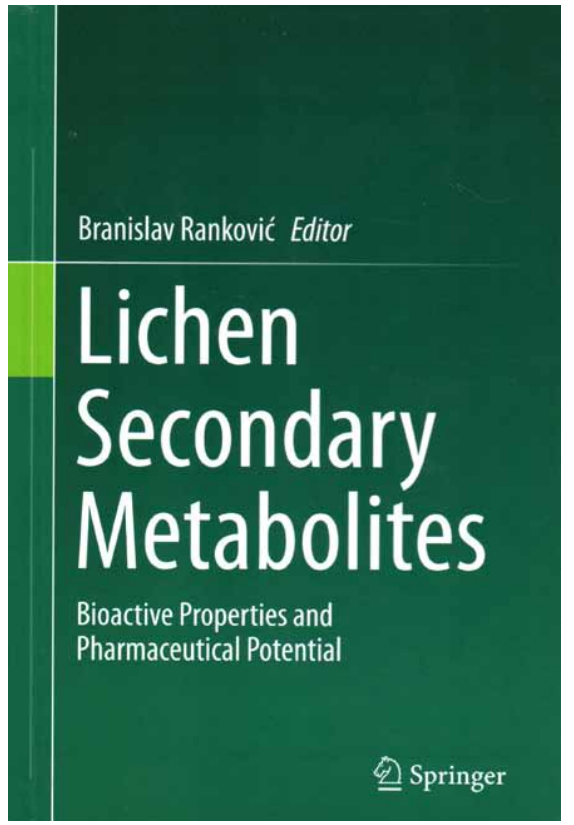

I was gratified to see this book as, with the current interest in the discovery of novel bioactive compounds, especially ones with pharmaceutical potential, there was a need to bring together what was known of the activity of lichen compounds. The huge range of polyketides of various kinds synthesised by the fungal components of lichens have not had the same profile in drug discovery as those produced by other fungi due to difficulties due to their slow growth, even though many are known to have antibacterial properties. New technology increasingly has the potential to overcome those issues through the possibilities afforded by genetic engineering as well as developments in methods of culturing these fungi. What has been lacking is a work to bring together what is known as a guide to future bioprospecting in lichens, and now we have it for the first time.

A large part of the book is occupied by a superb and surely exhaustive survey of traditional uses of lichens in medicine, drawing together data from an extraordinarily dispersed ethnobiological literature. This is supplemented by a series of chapters on different applications: antimicrobials, antioxidants, anti-cancer, anti-toxic, antigenotoxic, and antiviral. There are also references to uses as analgesics, pesticides, and anti-inflammatory agents. I found the range of cancers against which activities had been found particularly encouraging.

This will be an extraordinarily valuable guide to possible lines of exploration and experimentation, but also a source of material to embellish lectures and field courses. While the whole is well-produced, however, it was strange to see no preface, nor a list of contributors, nor any index. The lack of an index will inevitably frustrate and restrict the ease with which reports of the activities of particular lichens can be located in the text. Nevertheless, the editor is to be congratulated in realising a clear vision and providing a work that will be of great value not only to lichenologists, but also to all those involved in all aspects of drug discovery.

Fossil Fungi. By Thomas N. Taylor, Michael Krings and Edith L. Taylor. 2015. ISBN 978-0-12-387731-4. Pp. xv + 382, figs 475 (mostly col.). Amsterdam: Academic Press. Price: US\$ 127.50.

Fossils are the record of evolution, and have the potential to provide hard evidence with which to date events and calibrate phylogenetic trees, as well as documenting past ecosystems and environments. Fungi are generally considered as rare in the fossil record, but this is perhaps at least in part due to the scarcity of palaeomycologists able to recognise and interpret them, and there have been some spectacular discoveries in the last $10-15$ years. There has also been a lack of a synthesis of what is known, which was much needed as mycologists too rarely cross paths with palaeontologists. No longer is there any excuse. Indeed, here is a beautifully well-written and illustrated authoritative work that should inspire more mycologists to look to the fossil record.

The authors are renowned palaeobotanists, and produced the standard text on the subject (Taylor et al. 2009), but Tom in particular has a long interest in fossil fungi and has discovered some that have come to prominence in the dating of nodes in phylogenetic trees, such as the Devonian Paleopyrenomycites devonicus. Following an introduction to the history of the study of fossil fungi from the early decades of the $19^{\text {th }}$ century, and an explanation of how fungi come to be fossilized, the authors speculate on the age of the kingdom - perhaps more than a billion years old. The principal phyla extant today may all have their origins a staggering 850-1100 Mya, but the poor representation in the fossil record leaves much room for speculation. There is little evidence of the cyanobacterial-fungal associations that might have been amongst the first colonizers of land, but there is a panoply of strange structures from the PreCambrian that have been considered fungal by some and many of which remain of uncertain affinity. Of these, quite a number have been suggested to be lichenized, but if so may well represent groups with no modern representatives - such as the

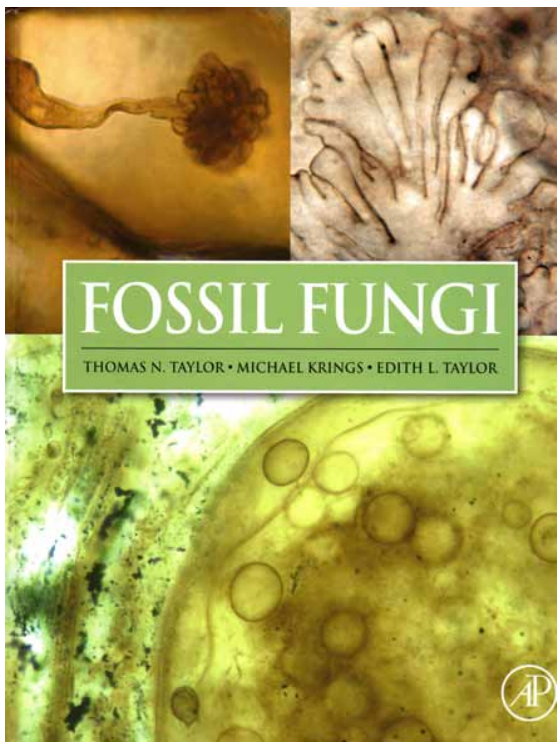

enigmatic massive Prototaxites, discussed in detail here, and on which the jury is still out.

The major part of the book comprises accounts of the fossil record of the various 
fungal phyla, all beautifully illustrated in colour with numerous previously unpublished photomicrographs by the authors and colleagues. There is a most valuable chapter on the naming of fossil fungal spores, including introductions to many of the generic names coined for these, which have generally ended with the suffix "-sporites". This serves as a valuable introduction to the compilation of fossil fungal spores by Kalgutkar \& Jansonius (2000). The penultimate chapter is a fascinating overview of fungal interactions, from coprolites and fungus gardens, to fungus-fungus and fungus-plant, the rhizosphere, and evidence for roles in rock weathering. Bacteria and fungus-like organisms feature in the last chapter where information on fossil slime moulds and straminipilous fungi is to be found.

With over 2400 titles in the reference list, this is a remarkable source-book for anyone concerned with or wishing to enter the field of palaeomycology. And if you wonder what some of the authors look like, the book has numerous (perhaps too numerous) photographs of those who have contributed to the field. For palaeontologists unfamiliar with mycospeak, there is also a glossary of some 700 terms. I was also pleased to see that there was a comprehensive index; the emerging trend of poor or no indices was fortunately not followed.
This will undoubtedly come to be recognized as one of the great books in mycology, and I cannot imagine any mycologist not finding something to intrigue and stimulate speculation within its pages.

Kalgutkar RM, Jansonius J (2000) Synopsis of Fossil Fungal Spores, Mycelia and Fructifications. [Contribution Series no. 39.] Dallas, TX: American Association of Stratigraphic Palynologists Foundation.

Taylor TN, Taylor EL, Krings M (2009)

Palaeobotany: the biology and evolution of fossil plants. $2^{\text {nd }}$ edn. Amsterdam: Academic Press.

Recent Advances in Lichenology. Edited by Dalip Kumar Upreti, Pradeep K. Divakar, Vertika Shukla, and Rajesh Bajpai. 2015.

Vol. 1. Modern methods and approaches in biomonitoring and bioprospection. ISBN 978-81-322-2180-7 (hdbk), 978-81-322-2181-4 (ebk). Pp. xv + 265, illustr. (some col.). Price: US\$ 239 (hdbk), US\$ 189 (ebk).

Vol. 2. Modern methods and approaches in lichen systematics and culture techniques. ISBN 978-81-322-2234-7 (hdbk), 978-8-322-2235-4 (ebk). Pp. xv + 232, illustr. (some col.). Price: US\$ 189 (hdbk), US\$ 149 (ebk).

New Delhi: Springer India.

Dalip Kumar Upreti · Pradeep K. Divakar Vertika Shukla · Rajesh Bajpai Editors

\section{Recent Advances in Lichenology}

Modern Methods and Approaches in Biomonitoring and Bioprospection, Volume 1

Springer

In a period of rapid progress in understanding, topical reviews of advances are to be welcomed as they can review an often disparate literature and place findings in a broader context. Critical reviews are sparse in lichenology, so this initiative
Dalip Kumar Upreti · Pradeep K. Divakar Vertika Shukla · Rajesh Bajpai Editors

\section{Recent Advances in Lichenology}

Modern Methods and Approaches in Lichen Systematics and Culture Techniques, Volume 2

Springer

by a team of Indian lichenologists is to be welcomed. The choice of topics to be included is necessarily eclectic, and it is unlikely that any two lichenologists would make the same selection.

The first volume comprises 11 chapters and the second 12. The first covers factors affecting growth rates and lichenometry (two chapters) and the use of lichens as bioindicators of pollution and disturbance, set in the context of the EU protocols on lichen sampling agreed by the EU last year, and also ones comparing methodologies and statistical methods (six chapters). I found the one on particulates especially comprehensive and informative, and the prospects of what might be done through hyperspectral remote sensing exciting, though far from exploitable at this stage. There is also a thorough treatment of the role of lichens in biodeterioration which has been much needed, especially in the light of issues over the extent to which they can be damaging.

Contrary to what might be expected from the title of the first volume, there is but a single chapter on lichen products, and that is confined to methods of detection rather than bioprospecting. Indeed, that contribution would have sat more comfortably in the second volume which has five chapters concerned with these compounds, their diversity, biological activities, including as anti-cancer agents, 
polyketide synthase genes involved in their production, and uses as dyes. Interestingly, there is not as much overlap as might have been anticipated with the other recent volume on lichen chemistry (Ranković 2015) from a different office of the same publishers, as that majors on applications (see above, p. (30)).

Two of the other topics covered in the second volume merit considerable attention, those on species delimitation and molecular phylogenetic and phylogenomic approaches in lichen systematics and evolution. Also considered are the origins of the lichen lifestyle as revealed in the fossil record, the use of high-throughput sequencing in studies of population biology, and the ecological services lichens provide. Two chapters are concerned with cultures, one on fungi isolated from macrolichens in Himalaya which are considered endolichenic (but frustratingly with no evidence they were growing inside the lichens), and the other on compounds produced in cultures of the isolated fungal partners.

The volumes are produced to a high standard, but as both are rather slim and there is overlap in content between them, a single volume might have been preferable. Four small things were rather irritating: the lack of an index to scientific names, the failure to use current scientific names throughout, the sporadic and unnecessary use of author citations of scientific names, and the lack of the volume or subtitle on the spine. An identical Preface appears at the start of each volume, but relates only to the first. There are many topics in lichenology in addition to those covered here that that would also merit topical reviews, and perhaps some of the points raised here could be borne in mind if a third volume is being contemplated by the editors.

Ranković B (2015) Lichen Secondary Metabolites: bioactive properties and pharmaceutical potential. Cham: Springer International Publishing. 